\title{
多溴二苯醚取代特征对其芳香烃受体结合能力的效应分析
}

\author{
姜龙 ${ }^{a, b}$ 程冰川 ${ }^{a, b}$ 李鱼*,a,b \\ ( ${ }^{a}$ 华北电力大学资源与环境研究院 北京 102206) \\ ( $b$ 华北电力大学区域能源系统优化教育部重点实验室 北京 102206)
}

\begin{abstract}
摘要 以已知 18 种多澳二苯醚(polybrominated diphenyl ethers, PBDEs)芳香烃受体结合能力实验值为因变量, 构建基于 取代基参数的 PBDEs 芳香烃受体结合能力定量构效关系模型以补足 PBDEs 芳香烃受体结合能力值, 借助全析因实验 的分析方法研究不同取代位置对 PBDEs 芳香烃受体结合能力的主效应及二阶交互效应, 并分别从总取代基数、两苯环 取代相似性、同一苯环取代基分布性综合研究 PBDEs 取代特征对芳香烃受体结合能力的影响规律. 研究表明: PBDEs 芳香烃受体结合能力受各取代位置主效应和二阶交互效应的显著影响, 邻位取代基可显著降低PBDEs 芳香烃受体结合 能力, 对位取代基则显著增强, 间位取代基主效应较弱，主要通过与邻对位取代基间的二阶交互效应影响 PBDEs 芳香 烃受体结合能力; 总取代基数、两苯环取代相似性与 PBDEs 芳香烃受体结合能力无显著相关性，而同一苯环上取代基 间分散性越大，同系物芳香烃受体结合能力越小.
\end{abstract}

关键词＼cjkstart取代基参数; 多溴二苯醚; 芳香烃受体结合能力; 定量构效关系模型; 全析因实验; 相似性分析

\section{Effect Analysis of Substituent Characteristics of PBDEs on Its Ah Receptor Binding Affinities}

\author{
Jiang, Long ${ }^{a, b}$ Cheng, Bingchuan ${ }^{a, b} \quad \mathrm{Li}, \mathrm{Yu}^{*, a, b}$
}

( ${ }^{a}$ Resources and Environmental Research Academy, North China Electric Power University, Beijing 102206, China)

$\left({ }^{b}\right.$ MOE Key Laboratory of Regional Energy Systems Optimization, North China Electric Power University, Beijing 102206, China)

\begin{abstract}
Based on the known experimental Ah receptor binding affinities of 18 kinds of polybrominated diphenyl ethers (PBDEs), the quantitative structure-activity relationships (QSAR) model for PBDEs' Ah receptor binding affinities was established via 13 substituent parameters (total number of substituent, substituent number in different position, substituent positional relationship parameters, substituent difference between two rings) to complement unknown binding affinities of other 191 PBDEs. Then, the full factorial experiment with 10 factors which correlated with each substituent position and 2 level $(0,1)$ was applied to analyze the main effect and second-order interaction effect of each substituent position on PBDEs' Ah receptor binding affinities. Meanwhile, different analysis methods were used for the views of the total number of substituent, the similarity of different phenyl ring in single congener and the distribution of substituents on single phenyl ring to expound the correlation between substituent characteristics and Ah receptor binding affinities of PBDEs comprehensively. The obtained results have shown that: PBDEs' Ah receptor binding affinities are significantly affected by the main effect and second-order interaction effect of substitution positions, especially, the ortho-substituents can weaken the PBDEs' Ah receptor binding affinities and para-substituents have the opposite effect. The order of the importance for different position is presented as: para $>$ ortho $>$ meta. The main effect of meta-substituent is small which often affects the Ah receptor binding affinities of PBDEs by representing the second-order interaction effects combined with ortho/para-substituent. For other substituent characteristics, the total number of substituent and the similarity of different phenyl ring in single congener cannot control the Ah receptor binding affinities of PBDEs effectively, but the more decentralized for substituents on single phenyl ring, the smaller Ah receptor binding affinities for PBDEs.
\end{abstract}

Keywords substituent parameters; polybrominated diphenyl ethers; Ah receptor binding affinity; quantitative structure-activity relationships; full factorial experiment; similarity analysis

\section{1 引言}

多溴二苯醚 (polybrominated diphenyl ethers,
PBDEs)是一类常见的澳代阻燃添加剂，广泛应用于纺 织品、电路板、塑料制品、电子产品和建材等产品

*E-mail: liyuxx8@hotmail.com; Tel.: 010-61773886

Received April 16, 2014; published May 27, 2014.

Supporting information for this article is available free of charge via the Internet at http://sioc-journal.cn.

Project supported by the National Science \& Technology Pillar Program in the Eleventh Five-Year Plan period (No. 2008BAC43B01) and the Fundamental Research Funds for the Central Universities in 2013 (No. JB2013146).

项目受国家 “十一五” 科技支撑项目(No. 2008BAC43B01)和 2013 中央高校基本科研业务专项资金(No. JB2013146)资助. 
中 $^{[1 \sim 3]}$. 由于 PBDEs 聚合物彼此间缺乏化学键作用, 其 化学性质较不稳定, 因此, PBDEs 聚合产品在生产、使 用和废弃的过程中都有可能向周围环境中释放 $\mathrm{PBDEs}^{[4 \sim 6]}$, 而 PBDEs 在环境中比较稳定, 难以通过物 理、化学或生物方式降解, 同时, PBDEs 可通过食物链 逐级放大，对高营养级生物造成毒性富集影响 ${ }^{[7]}$.

除了在环境中具有分布广泛和持久性污染特性外, PBDEs 结构与其他有机卤代物相类似, 例如多氯联苯 (polychlorinated biphenyl, PCBs)、二噁英等, 因此逐渐引 起人们对 PBDEs 的潜在内分泌干扰活性和生物毒性的 探究 ${ }^{[8]}$. 研究表明, 大多数卤代碳氢化合物(halogenated hydrocarbons, HAHs)的生物毒性由芳香烃受体(aryl hydrocarbon receptor, AhR)调节, 一种通常情况下无活性 而与一些共分子伴侣胞质溶胶结合的转录因子, 属于基 螺旋-环-螺旋(Basic Helix-Loop-Helix/Per Arnt-Sim)蛋 白. 一旦 AhR 结合到 HAHs 等化学物质上时, 会引起分 子伴侣离解并导致 $\mathrm{AhR}$ 转移到细胞核中, 致使基因转 录发生改变, 对生物体造成严重危害 ${ }^{[9,10]}$, 而卤代芳香 化合物与 AhR 的结合能力(Ah receptor binding affinities) 和其生物毒性呈正相关性, 因此芳香烃受体结合能力的 大小被广泛用来表征有机物生物毒性的大小程度 ${ }^{[11,12]}$. 相比 PCBs 与二啞英, 有关 PBDEs 芳香烃受体结合能力 研究的文献报道较少, 对 PBDEs 芳香烃受体结合机理 认识也较为模糊, 研究重点多集中于 PBDEs 自身各同 系物芳香烃受体结合能力预测及与其余卤代物间的比 较. 对于相同的芳香烃受体结合能力实验数据源 ${ }^{[13]}$, 研 究者分别采用比较分子场方法 ${ }^{[14,15]}$ 、支持向量机 ${ }^{[16]}$ 、多

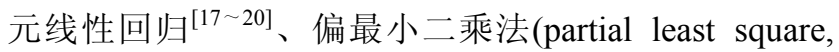
PLS) $)^{[21]}$ 等方法构建 PBDEs 芳香烃受体结合能力定量构 效关系模型(quantitative structure-activity relationships, QSAR), 研究的核心内容围绕利用不同种参数类型提高 QSAR 模型的性能, 并通过比较分析所建模型性能优劣, 但所涉及拓扑或量化参数物理化学意义抽象, 不易开展 可控研究.

取代基参数不同于量化、拓扑等类型参数, 其反映 的即为在各个取代位置上的取代基个数或特征, 通过研 究和总结取代基参数与 PBDEs 芳香烃受体结合能力间 的作用关系, 可以直观的寻求调控 PBDEs 芳香烃受体 结合能力的取代特征. $\mathrm{Gu}$ 等 ${ }^{[21]}$ 研究表明显著影响 PBDEs 芳香烃受体结合能力的量化参数 $\Delta \alpha$ 和取代位置 相关, 即间位 $\left(3,3^{\prime}\right)$ 与对位 $\left(4,4^{\prime}\right)$ 溴取代基可增强 PBDEs 的结合能力, 邻位 $\left(2,2^{\prime}\right)$ 与间位 $\left(5,5^{\prime}\right)$ 溴取代基可减弱 PBDEs 结合能力, 相同的研究结论也在文献[19]中提及, 而 Luthe ${ }^{[22]}$ 和龙杰义 ${ }^{[23]}$ 在对 PCBs 芳香烃受体结合能力 研究中同样得出 3,4 位取代基可以增强结合能力, 邻位 取代可降低其结合能力. 但是, 过往对取代特征的研究 仅限于在芳香烃受体结合机理研究中的扩展性分析, 且 只针对单个取代位置对 PBDEs 芳香烃受体结合能力的
作用规律，对于位置间的交互效应及分子整体的取代特 征并未涉及, 而决定 PBDEs 芳香烃受体结合能力不仅 仅是单一位置的作用结果.

析因实验在实验设计中被广泛地用来准确评估各 实验因素对目标检测量的单独影响程度(主效应)及彼此 交互产生的联合影响程度(各阶交互作用)大小，例如李 鱼等 ${ }^{[24]}$ 利用析因实验设计研究了重金属及农药共存下 各物质浓度对目标污染物吸附的主效应及二阶交互效 应，但尚未被应用到取代特征的理论分析中. 析因设计 所需实验次数多, 一般而言, 无论是在体内还是体外开 展生物测定实验都是比较耗时，同时花费巨大，而 QSAR 模型是对实验缺失值进行补充的重要途径. 本文 基于已检测的 PBDEs 芳香烃受体结合能力实验数据, 通过 QSAR 模型的构建实现对其余同系物芳香烃受体 结合能力的数据补足, 将全析因设计的思想合理转化应 用于 PBDEs 各取代位置, 并结合 QSAR 模型所得芳香 烃受体结合能力数据, 分析各取代位置对 PBDEs 芳香 烃受体结合能力的主效应及二阶交互效应，同时分别从 总取代基数、两苯环取代相似性、同一苯环取代基分布 性综合研究取代特征对 PBDEs 芳香烃受体结合能力的 影响规律，从而在避免大量的实验工作同时实现基于取 代特征影响规律的 PBDEs 芳香烃受体结合能力调控.

\section{2 结果与讨论}

\section{1 基于取代基参数的 PBDEs 芳香烃受体结合能力 QSAR 模型构建}

以 PBDEs 与芳香烃受体的相对结合能力(relative binding affinities, RBA)的负对数值作为因变量, 对建模 组数据进行 PLS 回归, 所得 PBDEs 芳香烃受体结合能 力 QSAR 预测模型及其参数如下所示:

$$
\begin{aligned}
& -\operatorname{logRBA}=4.492-0.086 N_{\mathrm{T}}+0.013 N_{2,6}-0.162 N_{2} \\
& +0.52 N_{6}-0.109 N_{3,5}-0.470 N_{3}+0.388 N_{5}-0.572 N_{4} \\
& +0.17 N_{o}+0.058 N_{m}+0.35 N_{p}-0.212 N_{D}+0.11 N_{2,4,6}
\end{aligned}
$$

其中: $R_{x}^{2}=0.904, R_{Y}^{2}=0.935, R^{2}=0.906, q^{2}=0.720$, $P R E S S=2.157, F=31.83, R_{\mathrm{pre}}^{2}=0.828$, 主成分数 $N$ 为 3 . 其中 $R_{x}^{2}$ 和 $R_{Y}^{2}$ 分别表示模型对自变量集和因变量集的解 释能力, 通过 $R^{2}$ 和 $R_{\mathrm{prc}}^{2}$ 说明模型(1)具有较好的拟合性和 预测性. 图 1 为 18 种已知芳香烃受体结合能力的 PBDEs 同系物 $-\log R B A$ 实验值和预测值间的比较, 绝 大部分点分布在斜率为 1 的直线上，表明各同系物芳香 烃受体结合能力预测值和计算值趋于相等, 偏差较大的 点对应的是 BDE-85, 而以往的研究也证实 QSAR 模型 对 BDE-85 芳香烃受体结合能力预测值和实验值的偏差 往往是数据集平均偏差的两倍以上 ${ }^{[19,21]} .209$ 种 PBDEs 同系物芳香烃受体结合能力预测数据详见附表 1(支持 信息). 由已有文献中 QSAR 模型预测所得 209 种 
PBDEs 芳香烃受体结合能力和本文模型所得预测值间 的对比图(附图 1, 支持信息)中点均分布在斜率为 1 直线 两侧，且本文所建模型预测值同文献[14]和 [18]的芳香 烃受体结合能力值间标准偏差 $(S D)$ 分别在 $0.01 \sim 0.49$ 和 $0 \sim 0.61$ 范围内, 与文献 [19]相比, 除 BDE-142、 BDE-150、BDE-166、BDE-198 预测值略偏低(SD 区间 为 $0.72 \sim 1.22$ ) 外, 其余大部分预测值 $\mathrm{SD}$ 区间为 $0.02 \sim$ 0.60 可知, 本文所建模型的预测值能较好反映其自身结 合能力大小.

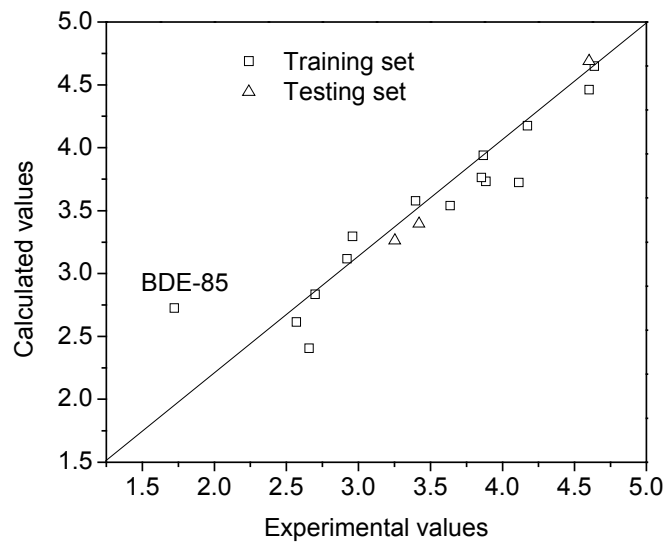

图 1 PBDEs 芳香烃受体结合能力实验值与预测值对比

Figure 1 Comparison between experimental and calculated values of $-\operatorname{logRBA}$ for PBDEs

\section{2 基于全析因实验的 PBDEs 取代位置效应分析}

\subsection{1 模型适合性检验}

图 2 为固定效应模型对 PBDEs 芳香烃受体结合能 力数据的适合性检验残差图, 图中模型残差的正态概率 图和直方图没有偏离正态分布的异常点出现, 表明方案
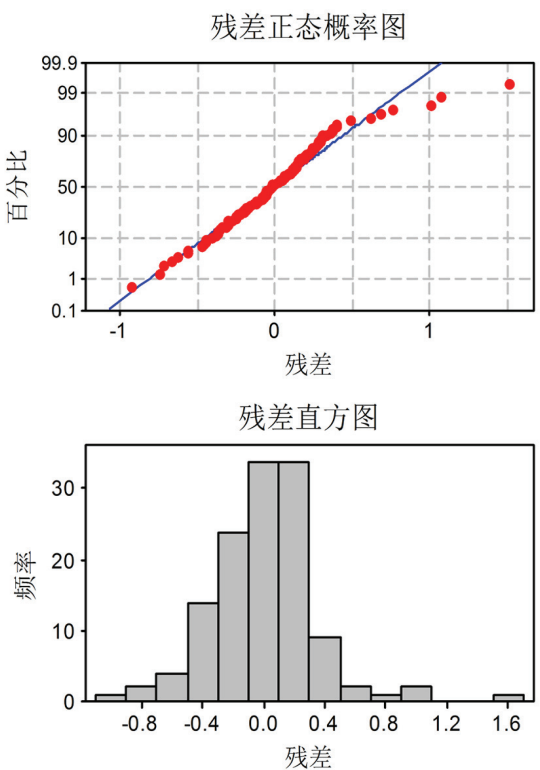

满足模型的正态性假设要求; 残差与拟合值的关系图, 未发现残差与拟合值有任何明显的模式，模型满足方差 的齐性假设; 依时间序列残差图没有出现正残差或负残 差的情况，表明模型不存在违反独立性的模式. 上述残 差分析表明使用固定效应模型可以进行主效应和二阶 交互效应分析 ${ }^{[24]}$.

\subsubsection{PBDEs 取代位置的效应分析}

表 1 为各取代位置对 PBDEs 芳香烃受体结合能力 的主效应及二阶交互效应方差分析表，主效应和二阶交 互效应 $P$ 值都远低于显著性水平 0.05 , 因此本文研究的 10 种因素的主效应和二阶交互效应整体上对 PBDEs 芳 香烃受体结合能力都有显著的影响，同比主效应对芳香 烃受体结合能力影响更大.

表 1 取代位置对 PBDEs 芳香烃受体结合能力主效应及二阶交互效 应方差分析

Table 1 Variance analysis of the main effects and second-order interaction effects of substituent positions on PBDEs' Ah receptor binding affinities

\begin{tabular}{ccccc}
\hline 来源 & 自由度 & 均方 & $F$ & $P($ sig. $)$ \\
\hline 主效应 & 10 & 41.93 & 20.03 & 0.000 \\
二阶交互效应 & 45 & 25.01 & 2.66 & 0.000 \\
残差 & 72 & 15.07 & & \\
合计 & 127 & 82.01 & & \\
\hline
\end{tabular}

图 3 为取代位置主效应及二阶交互效应的标准化效 应正态分布图，距离拟合线距离越远的参数，其效应越 强，对应取代位置重要性越大，图中用红色标识; 不重 要参数的效应往往较小且分布在零值附近, 图中用黑色 标识. 图 3 中的重要主效应及二阶交互效应位置参数的
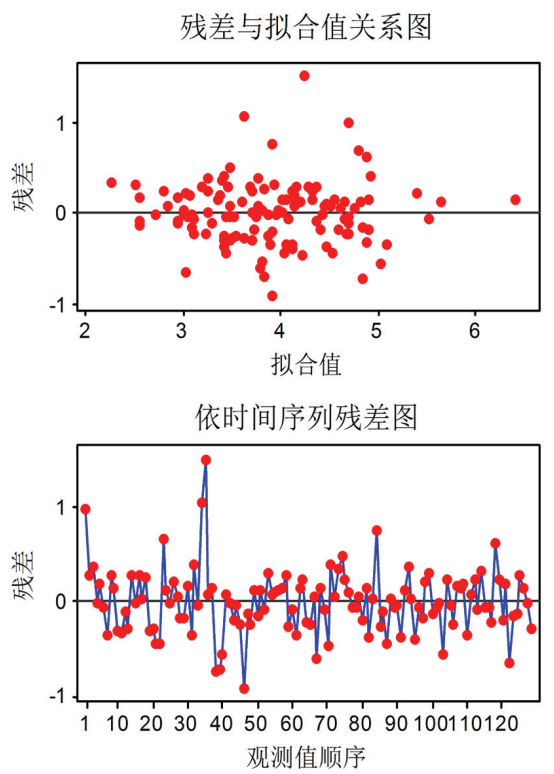

图 2 适合性检验残差图

Figure 2 Fit testing residual plots 


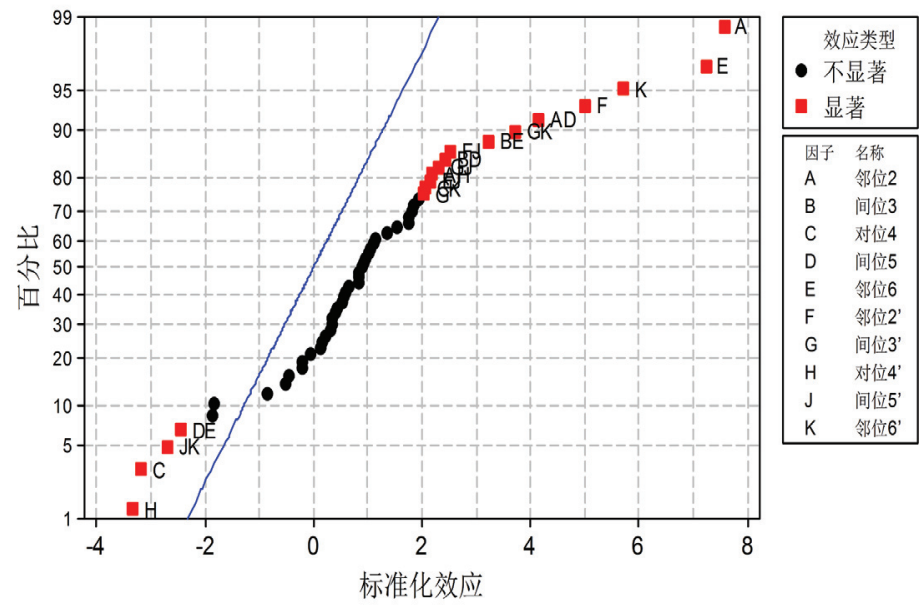

图 3 标准化效应正态分布图

Figure 3 Normal distribution of standardized effects

具体效应值在表 2 及表 3 中列举出来, 全部参数的效应 值分析见附表 2(支持信息).

(1) PBDEs 取代位置的主效应分析

表 2 为基于全析因实验所得取代位置主效应估计值 表, 邻位 $2 / 2^{\prime}$ 和邻位 $6 / 6^{\prime}$ 的效应估计值相近且皆显著正 相关于 $-\operatorname{logRBA}$, 即邻位溴取代基可降低 PBDEs 芳香 烃受体结合能力; 间位 $3 / 3^{\prime}$ 和间位 $5 / 5^{\prime}$ 的效应估计值相 近但与 PBDEs 芳香烃受体结合能力值间不具有显著相 关性; 对位取代 4/4'则可显著提高 PBDEs 芳香烃受体结 合能力. Wang ${ }^{[14]}$ 和 $\mathrm{Gu}^{[15]}$ 针对于和本文相同数据源分别 构建了 PBDEs 芳香烃受体结合能力 3D-QSAR 模型，从 文献[14]中的图 4 及文献[15]中的图 4/图 5 可以看出, 图 中的绿色部分即 $2,2^{\prime}, 5,5^{\prime}, 6,6^{\prime}$ 处表示此处加入溴取代基 将有利于 $-\log \mathrm{RBA}$ 值的提升, 即减弱同系物的芳香烃 受体结合能力, 4,4'为红色部分即加强同系物的芳香烃 受体结合能力. 结合显著性 P 值可知影响作用排序为邻 位 $>$ 对位 $>$ 间位, 邻对位取代位置的主效应显著相关于 PBDEs 芳香烃受体结合能力.

表 2 取代位置主效应估计值表

Table 2 Main effect values of substituent positions

\begin{tabular}{ccccc}
\hline \multirow{2}{*}{ 因素 } & 效应估计值 & \multicolumn{2}{c}{ 贡献率\% } & \multirow{2}{*}{$P(\mathrm{sig})}$. \\
\cline { 3 - 4 } 邻位 2,2' $(\mathrm{A}, \mathrm{F})$ & & + & - & \\
间位 3,3'(B, G) & 0.019 & 32.65 & & $(0.000,0.000)$ \\
对位 4,4' $(\mathrm{C}, \mathrm{H})$ & -0.526 & 7.40 & & $(0.411,0.046)$ \\
间位 5,5'(D, J) & 0.297 & 9.52 & & $(0.068,0.073)$ \\
邻位 6,6' $(\mathrm{E}, \mathrm{K})$ & 1.048 & 33.58 & & $(0.000,0.000)$ \\
邻位 & 2.067 & 66.23 & & \\
间位 & 0.528 & 16.92 & & \\
对位 & -0.526 & & 16.85 & \\
Total & 3.121 & 83.15 & 16.85 & \\
\hline
\end{tabular}

(2) PBDEs 取代位置的二阶交互效应分析

图 4 为取代位置二阶交互效应图，图中平行线表示 相应两参数间不存在交互作用，而线与线间斜率差别越 大，交互作用程度越高.

表 3 分析并列举了满足统计学上显著性要求的二阶 交互效应具体效应值，具有显著性的交互作用都有间位 参与，而间位主效应并不显著，可知间位取代往往在已 知邻对位取代情况下可以显著调控 PBDEs 同系物的芳 香烃受体结合能力.

表 3 二阶交互作用效应估计值

Table 3 Effect values of second-order interactions

\begin{tabular}{|c|c|c|c|c|}
\hline \multirow{2}{*}{ 因素 } & \multirow{2}{*}{ 效应估计值 } & \multicolumn{2}{|c|}{ 贡献率\% } & \multirow{2}{*}{$P$ (sig.) } \\
\hline & & + & - & \\
\hline 邻位 2, 间位 5(AD) & 0.336 & 13.86 & & 0.000 \\
\hline 邻位 2, 对位 4'(AH) & 0.177 & 7.30 & & 0.032 \\
\hline 间位 3, 间位 5(BD) & 0.198 & 8.17 & & 0.017 \\
\hline 间位 3, 邻位 6(BE) & 0.262 & 10.81 & & 0.002 \\
\hline 对位 4, 邻位 6'(CK) & 0.167 & 6.89 & & 0.043 \\
\hline 间位 5, 邻位 6(DE) & -0.198 & & 8.17 & 0.017 \\
\hline 邻位 6, 间位 5'(EJ) & 0.174 & 7.18 & & 0.035 \\
\hline 邻位 $2^{\prime}$, 间位 $5^{\prime}(\mathrm{FJ})$ & 0.205 & 8.46 & & 0.013 \\
\hline 间位 3'，间位 5'(GJ) & 0.187 & 7.71 & & 0.023 \\
\hline 间位 3', 邻位 6'(GK) & 0.302 & 12.46 & & 0.000 \\
\hline 间位 5', 邻位 6'(JK) & -0.218 & & 8.99 & 0.009 \\
\hline $\mathrm{AD}+\mathrm{FJ}$ & 0.541 & 22.32 & & \\
\hline $\mathrm{BD}+\mathrm{GJ}$ & 0.385 & 15.88 & & \\
\hline $\mathrm{BE}+\mathrm{GK}$ & 0.564 & 23.27 & & \\
\hline $\mathrm{DE}+\mathrm{JK}$ & -0.416 & & 17.16 & \\
\hline Total & 2.424 & 82.84 & 17.16 & \\
\hline
\end{tabular}




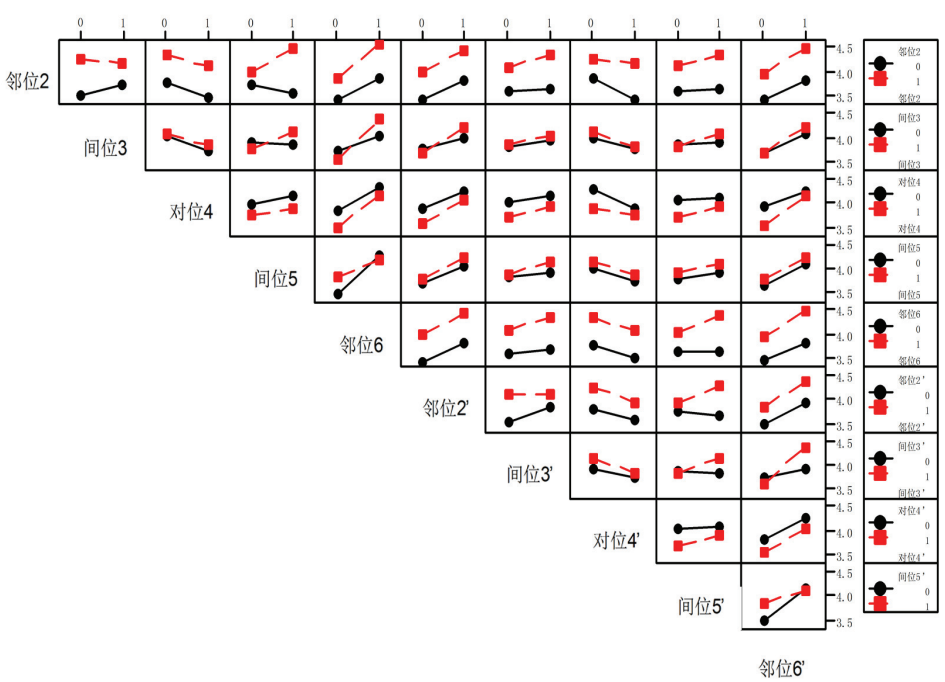

图 4 取代位置二阶交互效应图

Figure 4 The second-order interaction effects of substituent positions

由图 4 可知，当 $2 / 2^{\prime}$ 位没有取代基时，5/5'位存在取 代基时芳香烃受体结合能力变大; 当 $2 / 2^{\prime}$ 位具有取代基 时, 5/5'位存在取代基时芳香烃受体结合能力显著变小, 由于 $2 / 2$ '位溴取代基的主效应是降低 PBDEs 芳香烃受体 结合能力, 可知 $5 / 5^{\prime}$ 位溴取代基的引入将会加强 $2 / 2$ '位 降低芳香烃受体结合能力的效应. 同时, 表 3 中 $\mathrm{AD}$ 和 FJ 显著正相关一 $\operatorname{logRBA}$ 即负相关于芳香烃受体结合能 力, 邻位 $2 / 2^{\prime}$ 和间位 $5 / 5^{\prime}$ 的溴代基组合数越大, 芳香烃受 体结合能力越小。例如： $(1,0,0,1,0,1,0,0,1,0)$ 即 BDE-52(5.328) 的芳香烃受体结合能力比 $(1,0,0,1,0,0,0,0,0,0)$ 即 BDE-9(4.486)小. 同时可以看出, $(1,0,0,1,0,0,0,0,0,0)$ 即 BDE-9 $(4,486)$ 的芳香烃受体结合能 力比 $(0,0,0,1,0,0,0,0,0,0) \mathrm{BDE}-2(3.615)$ 小, 这也和图 4 中 的交互作用规律相符.

对于 $\mathrm{DE}$ 和 $\mathrm{JK}$ 的二阶交互效应而言, 当间位 $5 / 5^{\prime}$ 与邻位 $6 / 6^{\prime}$ 同时未取代时, 同系物芳香烃受体结合能力 较大; 邻位 6/6'有溴取代基时, 间位 5/5'有无取代基芳香 烃受体结合能力变化不大, 结合能力均较低; 间位 $5 / 5^{\prime}$ 取代基的引入总趋势是降低同系物芳香烃受体结合能 力, 有无邻位 $6 / 6^{\prime}$ 取代时所降低的程度不同. 由表 3 中 $\mathrm{DE}$ 和 JK 显著负相关于 $-\operatorname{logRBA}$ 即正相关于芳香烃受 体结合能力的规律可知, 对于 5,6 位同时取代时, 邻位 $6 / 6^{\prime}$ 和间位 $5 / 5^{\prime}$ 的溴代基组合数越大, 生物毒性越大, 即 BDE-40 $(0,0,0,1,1,0,0,0,1,1)$ 的芳香烃受体结合能力 (3.252)大于 BDE-5 $(0,0,0,1,1,0,0,0,0,0)$ 的芳香烃受体结合 能力(3.448), 亦或 BDE-82 $(0,0,1,1,1,0,0,0,1,1)$ 的芳香烃 受体结合能力 (2.72) > BDE-21 $(0,0,1,1,1,0,0,0,0,0)$ 的芳香 烃受体结合能力 (3.224) $>$ BDE-3 $(0,0,1,0,0,0,0,0,0,0)$ 的芳 香烃受体结合能力(3.732).

由图 4 可知, BD、BE、GJ、GK 四种二阶交互效应 规律变化一致, 即 $(3,5) 、(3,6) 、\left(3^{\prime}, 5^{\prime}\right) 、\left(3^{\prime}, 6^{\prime}\right)$ 中两位置同
时发生溴取代时对应的同系物芳香烃受体结合能力最 小, 且上述二阶交互效应估计值皆为正值, 即正相关于 $-\operatorname{logRBA}$ 值，负相关于芳香烃受体结合能力，可知间 位 $3 / 3^{\prime}$ 和间位 $5 / 5^{\prime}$ 的溴代基组合数及间位 $3 / 3^{\prime}$ 和邻位 $6 / 6^{\prime}$ 越大，芳香烃受体结合能力越小，例如: $(0,1,0,1,0,0,1,0,1,0)$ 即 BDE-80(3.664) 比 $(0,1,0,1,0,0,0$, 0,0,0)BDE-14(3.654) 小; BDE-101 $(0,1,1,0,1,0,1,1,0,1)$ 的 芳香烃受体结合能力 (4.796) $<$ BDE- $29(0,1,1,0,1,0,0,0$, $0,0)$ 的芳香烃受体结合能力 (3.954) $<$ BDE-3 $(0,0,1,0,0$, $0,0,0,0,0)$ 的芳香烃受体结合能力(3.732).

其余与 PBDEs 芳香烃受体结合能力显著相关的 3 种二阶交互效应皆涉及到两个苯环同时取代的情况: 邻 位 2 和对位 $4^{\prime}(\mathrm{AH})$ ，对位 4 和邻位 $6^{\prime}(\mathrm{CK})$ 、邻位 6 和间 位 $5^{\prime}(\mathrm{EJ})$, 且均与 $-\log R B A$ 显著正相关, 即可明显降低 PBDEs 芳香烃受体结合能力.

\section{3 取代基个数与 PBDEs 芳香烃受体结合能力相关 性分析}

以往研究所构建基于相同数据源的 PBDEs 芳香烃 受体结合能力 QSAR 预测模型采用的是不同类型参数 和建模方法 ${ }^{[14 ~ 21]}$, 由其所获得各同系物 - $\operatorname{logRBA}$ 预测 值绘制不同溴数取代基的芳香烃受体结合能力分布如 图 5 所示, 其中横线为均值, 各个 QSAR 模型预测出的 PBDEs 同系物 $-\log$ RBA 均值和总取代基数都不具有显 著的线性相关性. 由于上述模型均未把取代基参数参与 建模, 故分析结果能客观的体现出溴取代基数和芳香烃 受体结合能力间相关性大小.

\section{4 两苯环取代相似性与 PBDEs 芳香烃受体结合能 力间相关性分析}

引入两环取代相似性的定量指标, 即以已知 18 种 实验检测 $-\log R B A$ 值的各同系物分子左苯环取代情况 

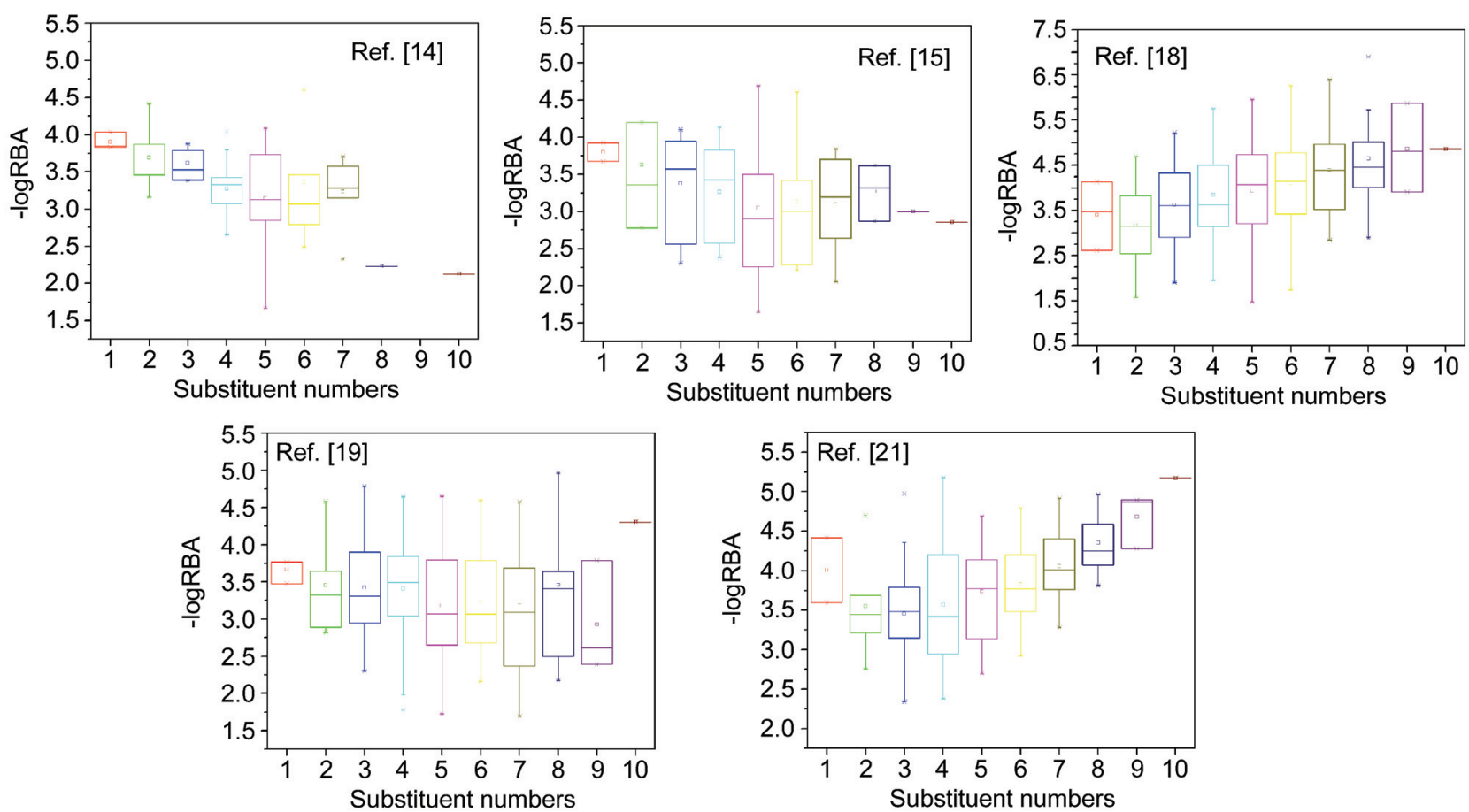

图 5 澳取代基数和 PBDEs 芳香烃受体结合能力分布区间图

Figure 5 Interval distribution between bromine-substituent numbers and - $\operatorname{logRBA}$ of PBDEs
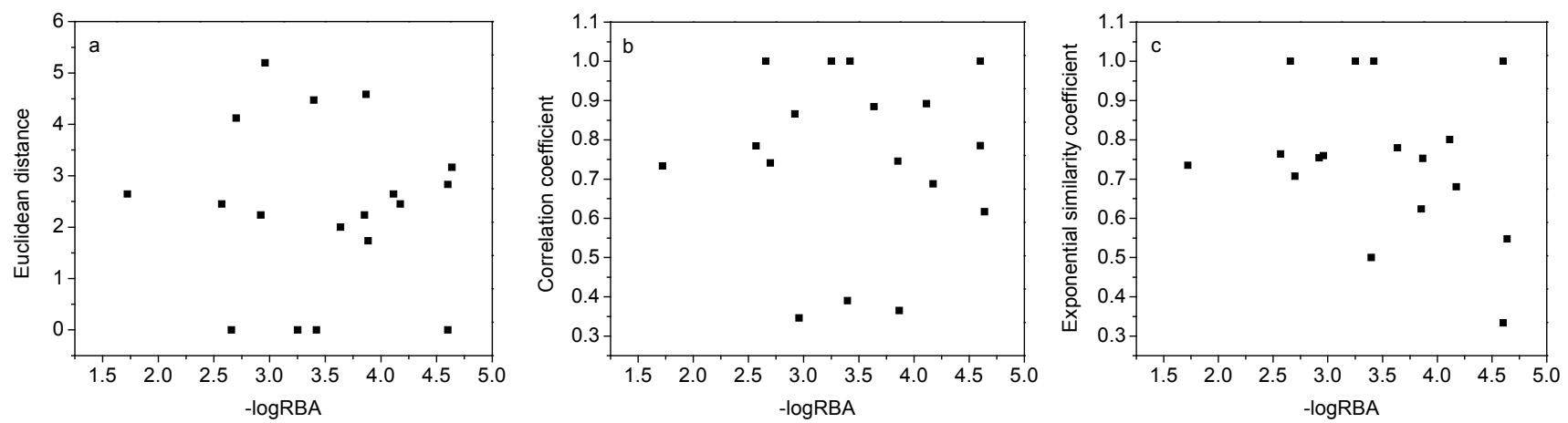

图 6 两苯环溴取代基相似性与芳香烃受体结合能力相关性分析( $\mathrm{a}$ 为欧式距离法, $\mathrm{b}$ 为相关系数法, $\mathrm{c}$ 为指数相似系数法)

Figure 6 Correlation analysis between the bromine-substituent similarities of two benzenes and - logRBA (a: Euclidean distance, b: Correlation coefficient, c: Exponential similarity coefficient)

为参照物, 分别选取距离测度、相似测度中的 3 种不同 的测试方法 (欧氏距离法、相关系数法、指数相似系数 法)对两苯环取代类型的相似性进行量化分析. 图 6 为 不同方法所得 18 种同系物取代相似性与各自 $-\log R B A$ 实验值相关分析图, 图中点分布分散, 即两苯环取代相 似性和 PBDEs 的芳香烃受体结合能力无显著相关性. 对于左右两苯环取代情况一致的 BDE-15、BDE-47、 BDE-77、BDE-153, 其- $\operatorname{logRBA}$ 值相差 1.94, 按照 Papa 等 ${ }^{[18]}$ 的芳香烃受体结合能力等级划分标准可知, BDE-77 具有中等芳香烃受体结合能力, 其余 3 种具有 低等芳香烃受体结合能力, 差距较大.

欧式距离表达式如下:

$$
O\left(X_{l}, X_{r}\right)=\sqrt{\sum_{i=1}^{n}\left(x_{l i}-x_{r i}\right)^{2}}
$$

相关系数如下式所示:

$$
S\left(X_{l}, X_{r}\right)=\frac{\sum_{i=1}^{n}\left(x_{l i}-\bar{x}_{l}\right)\left(x_{r i}-\bar{x}_{r}\right)}{\sqrt{\sum_{i=1}^{n}\left(x_{l i}-\bar{x}_{l}\right)^{2} \sum_{i=1}^{n}\left(x_{r i}-\bar{x}_{r}\right)^{2}}}
$$

指数相似系数: 


$$
e\left(X_{l}, X_{r}\right)=\frac{1}{n} \sum_{i=1}^{n} \exp \left[-\frac{3}{4} \frac{\left(x_{l i}-x_{r i}\right)^{2}}{\sigma_{i}^{2}}\right]
$$

其中 $X_{1}$ 和 $X_{\mathrm{r}}$ 分别为左右两苯环取代基参数构成的模式 向量, $\bar{x}_{l}$ 和 $\bar{x}_{r}$ 分别为模式向量 $X_{1}$ 和 $X_{\mathrm{r}}$ 的平均值 ${ }^{[25]}$.

\section{5 单一苯环取代基分散性与 PBDEs 芳香烃受体结 合能力相关性分析}

采用偏最小二乘法提供的变量投影重要性(VIP)来 分析同一苯环取代基间位置关系 $\left(N_{p} 、 N_{m} 、 N_{o}\right)$ 与 PBDEs 芳香烃受体结合能力的相关性, 其中 VIP $>1$ 所对应的 参数对芳香烃受体结合能力具有显著的影响能力 ${ }^{[26]}$, 结合相关性分析所得数值符号正负判别各参数对芳香 烃受体结合能力所起的抑制/促进作用, 结果如表 4 所 示.

\section{表 4 各取代基参数 VIP 值和相关性分析}

Table 4 VIP value and correlation analysis of each substituent parameter

\begin{tabular}{cccc}
\hline 变量 & $N_{o}$ & $N_{m}$ & $N_{p}$ \\
\hline VIP & 0.68 & 0.94 & 1.23 \\
相关性 & + & + & + \\
\hline
\end{tabular}

由表 4 可知, 三者皆正相关于 $-\operatorname{logRBA}$ 即负相关 于 PBDEs 芳香烃受体结合能力, 只有 $N_{p}$ 的 VIP 值大于 1 即显著负相关于 PBDEs 的芳香烃受体结合能力, 且影 响大小顺序为: $N_{p}>N_{m}>N_{o}$. 较邻位和间位分布而言, 对位分布的取代基在同一苯环上溴取代基彼此间距离 更远即分散性更大, 且不同于邻位或间位分布往往造成 吸电子的溴取代基集中在苯环的一侧所造成的负电荷 集中分布, 对位分布往往导致具有负电荷的溴取代基分 布苯环两侧, 造成苯环电荷密度分布更为均匀, 因此由 $N_{p}$ 负相关于芳香烃受体结合能力可知, 同一苯环内取 代基分散性越好, 苯环电荷密度分布差异越小, 芳香烃 受体结合能力越小. 在已知芳香烃受体结合能力实验数 据的 PBDEs 同系物中, 对于溴取代基数小于 4 的 10 种 PBDEs 同系物而言, 只有 BDE-49 的 $N_{p}$ 值为 1 (其余皆为 0 ), 其 $-\log R B A$ 值为 4.17 , 为其中唯一大于 4 的同系物.

\section{3 结论}

由取代基参数所构建的 PBDEs 芳香烃受体结合能 力 $\mathrm{QSAR}$ 方程的 $R^{2}$ 与 $R_{\mathrm{pre}}^{2}$ 值分别为 0.906 和 0.828 , 表 明方程对于 PBDEs 芳香烃受体结合能力值具有较好的 拟合性和预测性. 基于上述所得基础数据分别从取代位 置、总取代基数、两苯环取代相似性、同一苯环取代基 分布性系统分析取代特征对 PBDEs 芳香烃受体结合能 力的影响规律. 其中, 不同取代位置对 PBDEs 芳香烃受 体结合能力的影响顺序为: 邻位 $>$ 对位 $>$ 间位, 邻位取 代基的引入可显著减弱 PBDEs 芳香烃受体结合能力,
对位取代基则显著提高，间位取代基主效应较弱，往往 通过和邻/对位的二阶交互作用调控 PBDEs 的芳香烃受 体结合能力; 总溴取代基数及两苯环间取代相似性均同 PBDEs 生芳香烃受体结合能力无显著相关性，而单一 苯环上的取代基分布越分散, 所对应的 PBDEs 同系物 的芳香烃受体结合能力越小.

\section{4 材料与方法}

\subsection{PBDEs 芳香烃受体结合能力实验数据}

本文所用 18 种 PBDEs 同系物的芳香烃受体结合能 力数据引自文献 [13]: 以四氯二苯并 $-p$-二噁英与 $\mathrm{AhR}$ 的 结合能力为标准值, Chen 选取了 18 种取代类型不同的 PBDEs 同系物作为目标污染物, 对其与大鼠肝细胞的 相对结合能力进行了检测, 即实际结合能力同标准值间 的比值, 为便于本文 QSAR 模型的建立与分析, 将文献 [13]表 2 中 PBDEs 的 RBA 值进行负对数处理, 即以 $-\operatorname{logRBA}$ 值作为最终构建模型的因变量, 同芳香烃受 体结合能力成反比, 单位无量纲.

\subsection{QSAR 模型建立}

QSAR建模所需溴取代基参数涵盖 4 方面取代特征. （1）总取代基数 $\left(N_{\mathrm{T}}\right)$; (2)不同邻间对位取代基个数: 邻位 取代基数 $\left(N_{2,6}, N_{2}, N_{6}\right)$, 间位取代基数 $\left(N_{3,5}, N_{3}, N_{5}\right)$, 对位 取代基数 $\left(N_{4}\right)$, 邻对位取代基数和 $\left(N_{2,4,6}\right)$; (3)两环取代基 数差 $\left(N_{D}\right)$; (4)单一苯环上取代基间位置关系: 取代基间 邻位对数 $\left(N_{o}\right)$ 、取代基间间位对数 $\left(N_{m}\right)$ 、取代基间对位 对数 $\left(N_{P}\right)$. PBDEs 不同取代基位置序号如图 7 所示.

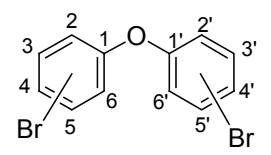

图 7 PBDEs 原子序号和分子结构示意图

Figure 7 Atom mark number and molecular geometry of PBDEs

以已知 PBDEs 的 $-\operatorname{logRBA}$ 值为模型因变量, 将 18 种 PBDEs 随机分为建模组和验证组(将已知 $-\log R B A$ 值的 PBDEs 同系物按照- $\operatorname{logRBA}$ 值大小排序, 同时为 了兼顾不同溴取代基数对结合能力的影响, 每 6 个同系 物中选取 1 个具有不同澳取代基数的代表性同系物作为 验证组样本), 借助于偏最小二乘法运算建模组数据构 建基于取代基参数的 PBDEs 芳香烃受体结合能力 QSAR 模型.

检验一个模型的有效性往往需要从拟合性, 稳健

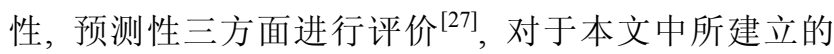
QSAR 模型, 分别从相关系数的平方 $\left(R^{2}\right)$ 、统计量 $\mathrm{F}$ 值 检测模型的拟合性; 从留一法交互检验系数 $\left(q^{2}\right)$ 、预测平 方和(PRESS) 对模型的稳健性进行评价, 若 $q^{2}>0.5$, 则 认为模型具有较好的稳健性 ${ }^{[28]}$; 对于最终所建 PBDEs 生物毒性 QSAR 模型从验证组所得数据间的相关系数 的平方 $\left(R_{\mathrm{pre}}^{2}\right)$ 对模型的预测性进行评价. 上述参数具体表 
达式如下所示 ${ }^{[16,19]}$ :

$$
\begin{aligned}
& R^{2}=\frac{\sum_{i=1}^{n}\left(\hat{y}_{i}-\bar{y}\right)^{2}}{\sum_{i=1}^{n}\left(y_{i}-\bar{y}\right)^{2}} \\
& F=\frac{\sum_{i=1}^{n}\left(\hat{y}_{i}-\bar{y}\right)^{2} / p}{\sum_{i=1}^{n}\left(y_{i}-\hat{y}_{i}\right)^{2} /(n-p-1)} \\
& \operatorname{PRESS}=\sum_{i=1}^{n}\left(y_{i}-\hat{y}_{i}\right)^{2} \\
& q^{2}=1-\operatorname{PRESS} / \sum_{i=1}^{n}\left(y_{i}-\bar{y}\right)^{2}
\end{aligned}
$$

其中, $y_{i}$ 为实验值, $\bar{y}$ 为实验值的平均值, $\hat{y}_{i}$ 为 QSAR 模型预测值, $n$ 为样本数 (预测组为 15 , 验证组为 3 ), $p$ 为 自变量个数. $R_{\mathrm{pre}}^{2}$ 公式同 $R^{2}$, 数据源由建模组数据变为验 证组数据即可.

\section{3 全析因实验设计}

将取代基位置作为全析因实验的因素，而每个因素 所对应的取代位置上的溴取代基数为水平. 根据 PBDEs 分子结构特征借由 Minitab 软件包中的实验设计 (design of experiment) 模块设计 10 因素即: 邻位 2(A)、 间位 3(B)、对位 4(C)、间位 5(D)、邻位 6(E)、邻位 $2^{\prime}(\mathrm{F})$ 、 间位 $3^{\prime}(\mathrm{G})$ 、对位 $4^{\prime}(\mathrm{H})$ 、间位 $5^{\prime}(\mathrm{G})$ 、邻位 $6^{\prime}(\mathrm{K})$ 两水平 $(0,1)$, 满足分析要求且实验量最小的分辨度 $V$ 的全析因实验 表, 括号内英文字母为 Minitab 软件中实际运算的各因 素表头代码(具体设计方案见附表 3, 支持信息), 不同位 置的取代搭配共有 $2^{10}$ 种组合方式即 1024 种取代类型, 每种取代类型对应于一种且唯一对应一种 PBDEs 同系 物. 而 PBDEs 和 DE 种类的总和仅为 210 种, 这是由于 PBDEs 分子两苯环间取代的对称性及单一苯环取代对 称性造成各同系物依各自相对对称性的高低, 分别有 $1 、 2 、 4 、 8$ 种取代类型这四类情况. 例如 $(1,0,0,0,0,0,0,0$, $0,0) 、(0,0,0,0,1,0,0,0,0,0) 、(0,0,0,0,0,1,0,0,0,0) 、(0,0,0,0,0$, $0,0,0,0,1) 4$ 种取代类型均对应 BDE-1.

由于设计方案中各因素水平都是可控的, 即都为固 定因子, 故应用 Minitab 软件包中固定效应模型对主效 应及二阶交互效应进行方差分析及效应分析(显著性水 平 sig. $=0.05)$, 但首先需要利用残差分析模块检验固定 效应模型对已有数据是否满足适用性要求: 正态性假 设、独立性假设和方差的齐次性假设.

上述基于分辨度 $\mathrm{V}$ 的全析因实验所涉及的取代基 参数类型为各个取代位置上的取代基数, 可精确分析单 个取代基主效应及取代位置间的二阶交互效应，但尚无
法涵盖其他取代特征对 PBDEs 芳香烃受体结合能力的 影响, 因此需要分别从总取代基数、两苯环取代相似性 及同一苯环上取代基分布性进一步分析，综合以上研究 实现全面的系统的取代特征对 PBDEs 芳香烃受体结合 能力的影响规律分析.

\section{References}

[1] Erickson, P. R.; Grandbois, M.; Arnold, W. A.; McNeill, K. Environ. Sci. Technol. 2012, 46, 8174.

[2] Labunska, I.; Harrad, S.; Santillo, D.; Johnston, P.; Brigden, K. Environ. Sci.: Processes Impacts 2013, 15, 503.

[3] Cao, H. J.; He, M. X.; Han, D. D.; Sun, Y. H.; Xie, J. Atmos. Environ. 2011, 45, 1525.

[4] Su, G. Y.; Yu, Y. J.; Liu, H. L.; Yu, H. X. Chinese J. Anal. Chem. 2013, 41, 754. (苏冠勇, 余益君, 刘红玲, 于红霞, 分析化学, 2013, 41, 754.)

[5] Wang, Z. Y.; Zhai, Z. C.; Wang, L. S.; Chen, J. L.; Kikuchi, O.; Watanabe, T. J. Mol. Struct.: Theochem 2004, 672, 97.

[6] Na, S.; Kim, M.; Paek, O.; Kim, Y. Chemosphere 2013, 90, 1736

[7] Wahl, M.; Lahni, B.; Guenther, R.; Kuch, B.; Yang, L.; Straehle, U.; Strack, S.; Weiss, C. Chemosphere 2008, 73(2), 209.

[8] Lilienthal, H.; Hack, A.; Roth-Harer, A.; Grande, S. W.; Talsness, C. E. Environ. Health Persp. 2006, 114, 194.

[9] Gu, Y. Z.; Hogenesch, J. B.; Bradfield, C. A. Ann. Rev. Pharmacol. Toxicol. 2000, 40, 519.

[10] Denison, M. S.; Pandini, A.; Nagy, S. R.; Baldwin, E. P.; Bonati, L. Chem. Biol. Interact. 2002, 141, 3 .

[11] Safe, S. H. Crit. Rex. Toxicol. 1990, 21, 51.

[12] Kovarich, S.; Papa, E.; Gramatica, P. J. Hazard. Mater. 2011, 190, 106.

[13] Chen, G. S.; Konstantinov, A. D.; Chittim, B. G.; Joyce, E. M.; Bols, N. C.; Bunce, N. J. Environ. Sci. Technol. 2001, 35, 3749.

[14] Wang, Y. W.; Liu, H. X.; Zhao, C. Y.; Liu, H. X.; Cai, Z. W.; Jiang, G. B. Environ. Sci. Technol. 2005, 39, 4961

[15] Gu, C. G.; Ju, X. H.; Jiang, X.; Yu, K.; Yang, S. G.; Sun, C. Ecotox. Environ. Safe. 2010, 73, 1470

[16] Zheng, G.; Xiao, M.; Lu, X. H. QSAR Comb. Sci. 2007, 26, 536.

[17] Xu, H. Y.; Zou, J. W.; Yu, Q. S.; Wang, Y. H.; Zhang, J. Y.; Jin, H. S. Chemosphere 2007, 66, 1998.

[18] Papa, E.; Kovarich, S.; Gramaticam, P. Chem. Res. Toxicol. 2010, $23,946$.

[19] Gu, C. G.; Ju, X. H.; Jiang, X.; Wang, F.; Yang, S. G.; Sun, C. SAR QSAR Environ. Res. 2009, 20, 287.

[20] Wang, Y. W.; Zhao, C. Y.; Ma, W. P.; Liu, H. X.; Wang, T.; Jiang, G. B. Chemosphere 2006, 64, 515.

[21] Gu, C. G.; Goodarzi, M.; Yang, X. L.; Bian, Y. R.; Cheng, S.; Xin, J. Toxicol. Lett. 2012, 208, 269.

[22] Luthe, G.; Jacobus, J. A.; Robertson, L. W. Environ. Toxicol. Pharmacol. 2008, 25, 202.

[23] Long, J. Y.; Yi, H. B.; Liu, X. K.; Wang, Y. F. Acta Chim. Sinica 2012, 70, 949. (龙杰义, 易海波, 刘星楷, 汪易飞, 化学学报, 2012, 70,949.)

[24] Li, Y.; Wang, M.; Zhang, C.; Gao, Q. J. Jilin Univ. 2013, 43, 1595. (李鱼, 王檬, 张琛, 高茜, 吉林大学学报, 2013, 43, 1595.)

[25] Cheng, Y. Y.; Chen, M. J.; Wu, Y. J. Acta Chim. Sinica 2002, 60, 2017. (程翼宇, 陈闽军, 吴永江, 化学学报, 2002, 60, 2017.)

[26] Li, Y.; Jiang, L.; Li, X. L.; Hu, Y.; Wen, J. Y. Chem. Res. Chin. Univ. 2013, 29, 568 .

[27] Gramatica, P. QSAR Comb. Sci. 2007, 26, 694.

[28] OECD(Organisation for Economic Co-Operation and Development). Guideline document on the validation of (quantitative) structure-activity relationship [(Q)SAR] models. ENV/JM/MONO(2007)2, 2007. 